\title{
Sex Differences in a Hypoxia Model of Preterm Brain Damage
}

\author{
SONIA R. MAYORAL, GHEZAL OMAR, AND ANNA A. PENN \\ Neuroscience Program [S.R.M.] and Department of Pediatrics [G.O., A.A.P.], Stanford University School of Medicine, Stanford, \\ California 94305
}

\begin{abstract}
Male sex is a well-established risk factor for poor neurodevelopmental outcome after premature birth. The mechanisms behind this sex-related difference are unknown. The damage associated with prematurity can be mimicked in rodents by prolonged exposure to sublethal postnatal hypoxia. This chronic hypoxia leads to anatomical changes in mice that strongly resemble the loss of volume, decreased myelination, and ventriculomegaly seen in preterm newborns. However, no sex differences have been previously noted in this rodent model. We hypothesized that sex comparisons in hypoxic mice would show sex-related differences in brain volume and white matter loss in response to the same degree of hypoxic insult. Mice were placed in chronic sublethal hypoxia from postnatal day 3-11. Cortical, hippocampal, and cerebellar volumes and myelination indices were measured. We found that the male hippocampus, normally larger than the female, undergoes a greater volume loss compared with females $(p<0.05)$. Myelination, generally greater in males, was significantly disrupted by hypoxia in neonatal male forebrain. These results support the use of this rodent model to investigate the basis of sex-related susceptibility to brain damage and develop new sex-based neuroprotective strategies. (Pediatr Res 66: 248-253, 2009)
\end{abstract}

$\mathrm{M}$ ore than 400,000 infants are born prematurely in the United States each year, with $20 \%$ of these being very preterm $(<32$ wk gestation) (1). Preterm birth can lead to abnormal brain development with subsequent physical, cognitive, and behavioral deficits $(2,3)$. Even late preterm birth (32-37 wk gestation) may have subtle neurologic consequences (4). The numbers of children born preterm and the numbers of survivors continues to rise along with the significant costs associated with their poor neurologic outcome (5).

Risk factors for these poor neurologic outcomes have been identified across multiple studies. Increased risk is independently associated with both bronchopulmonary dysplasia (BPD) and male sex $(3,6)$. The biology that underlies these risk factors is poorly understood. Male susceptibility is of particular interest because it implies that there may be hormonal factors that, if better understood, might provide new avenues in neuroprotective strategies.

Received January 16, 2009; accepted April 21, 2009.

Correspondence: Anna A. Penn, MD, PhD, 300 Pasteur Dr., Grant Bldg. S228, Stanford, CA 94305; e-mail: apenn@stanford.edu

Supported by NIH Grant K08 NS044989, John Merck Fund for Developmental Disabilities, Susman \& Asher Foundation, and Packard Children's Health Initiative Funds (to A.A.P.); Grant T32 MH020016-09 from NIH Training grant (to S.R.M.).
Neuroimaging has demonstrated specific reductions in brain volume (age corrected) in the cortex, hippocampus, and cerebellum plus ventriculomegaly, particularly in male children with history of BPD after preterm birth $(6,7)$. These deficits, particularly in the hippocampus and cerebellum, often correlate with later cognitive deficits (8). Although longitudinal studies on human preterm infants suggest factors that correlate with damage, rapid progress requires animal models in which damage can be mimicked, physical and behavioral changes can be assessed, mechanisms can be unraveled, and interventions can be stringently tested.

In the past decade, chronic sublethal hypoxia treatment of neonatal rodents has been developed as a model of BPDrelated preterm brain damage (9-12). Brain development in newborn rodents during the first two postnatal weeks is similar to that seen in very preterm infants: cortical neurogensis is complete, hippocampal and cerebellar neurogenesis are robust, and synaptogenesis and myelination are beginning (13). Pups treated with sublethal hypoxia during this period have reduced brain volumes and ventriculomegaly, highly reminiscent of that seen in human preterm infants (9-12). In neonatal mice acutely examined at multiple times during a week of sublethal hypoxia, cell loss occurs and synaptogenesis is perturbed (9). Mice that are allowed to recover have a temporary increase in neurogenesis, but neither normal neuronal number nor the appropriate balance of excitatory to inhibitory neurons is regained (14-16). However, no sex differences have previously been reported in any neonatal rodent chronic hypoxia model.

We sought to establish whether there was a detectable sex-linked difference in neurologic damage in this rodent hypoxia model. Using unbiased volumetric techniques, we compared specific brain regions in male and female mouse pups undergoing normoxia $\left(21 \% \mathrm{O}_{2}\right)$ or hypoxia $\left(10 \% \mathrm{O}_{2}\right)$ from postnatal day $3-11$, quantified the extent of myelination, and determined hippocampal cell proliferation in both sexes.

\section{MATERIALS AND METHODS}

Hypoxia. All experiments complied with approved animal protocols. Hypoxia treatment was carried out as described in (9), with minor modifications. Treatment initiation at P3 with assessment at P11 was chosen to span the period of brain development most closely matched to human preterm

Abbreviations: BGII, Black Gold II; pHH3, phospho-Histone H3 
infants, to maximize pup survival and to allow comparison to prior reports (9) Sixteen C57BL/6 litters per round of experiments were culled to 4 male and 4 female pups at postnatal day 1 (P1). An additional lactating dam (CD-1 genotype) was introduced to improve pup survival, given the poor maternal behavior of C57BL/6 dams. On P3, half of the litters were placed in a hypoxic chamber. Chamber oxygen levels were reduced to $10 \%$ by means of an oxygen controller (Biospherix) that displaces oxygen with nitrogen gas. Control litters were kept in normoxic (21\% oxygen) conditions. Animals were maintained in hypoxia or normoxia for $8 \mathrm{~d}$. On P11, brains were collected and fixed in $4 \%$ paraformaldehyde for volumetric measurement. Tail clippings were taken to confirm sex via PCR genotyping.

PCR genotyping. DNA was extracted from tail clippings by proteinase $\mathrm{K}$ digestion (17). Sex was determined by PCR (18) using specific primers to the smcx and smcy genes [SMCXYF: 5'CCGCTGCCAAATTCTTTGG3' and SMCXYR: 5'TGAAGCTTTTGGCTTTGAG3'].

Brain sectioning. Parafomaldehyde fixed brains were sunk in 30\% sucrose for $2 \mathrm{~d}$ before sagittal sectioning at $50 \mu \mathrm{m}$ on a sliding microtome. Every 10th section cut throughout one hemisphere was collected onto a slide.

Volumetric analysis. Sections were Cresyl violet stained and imaged using a Leica DFC280 camera on a Leica stereoscope. Cortical, hippocampal, and cerebellar volumes were measured using the Cavalieri method as described (19). A point grid was placed over the images, and the number of points transecting the region of interest was counted. The estimated volume of each region of interest was calculated using the following expression: $V=T \times$ $a / p \times \sum P_{i}$, where $T=$ the distance between sections; $a / p=$ the area associated with each point on the grid; and $P_{i}=$ the number of points intersecting a brain region of interest. The $a / p$ is calculated by dividing the square of the distance between points on the grid $(\delta x)$ by the linear magnification of the images $(M)$. Therefore, $a / p=\delta x^{2} / M^{2}$. Estimated volumes were normalized to control (Fig. 1) or control male values (Fig. 2). The $p$ values were calculated using two-way ANOVA with Bonferroni post hoc comparison. All measurements were done blind to sex and treatment.

Myelin staining. Black Gold II [BGII; Histo-Chem, Inc; (20)] was used to reveal myelin. Sections were slide mounted, dried and rehydrated in distilled water for $2 \mathrm{~min}$, placed in $0.3 \%$ solution of BGII in $0.9 \%$ saline at $65^{\circ} \mathrm{C}$ for $12 \mathrm{~min}$, rinsed in distilled water for 2 min, placed in $1 \%$ sodium thiosulfate for $3 \mathrm{~min}$, washed three times in water, and finally dehydrated and cleared through ethanol and xylene. Bright field images of the myelin staining were

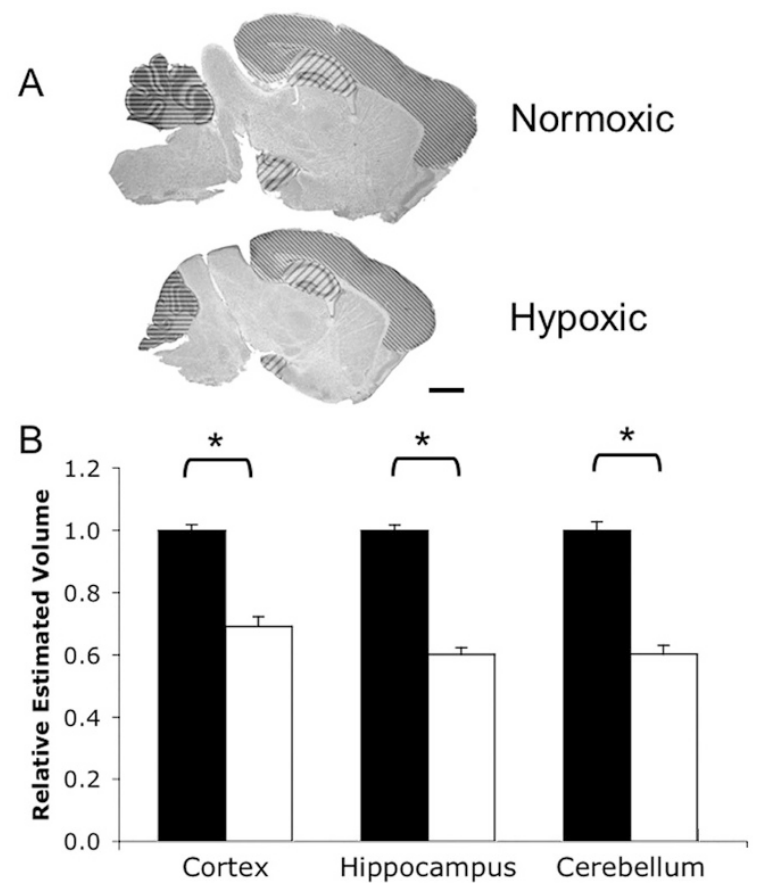

Figure 1. Chronic neonatal hypoxia reduces regional brain volumes. (A) Cresyl violet staining of normoxic and hypoxic brains of C57BL/6 mice at P11 showing hypoxia-associated size reduction, similar to rat (10). Regions measured are marked. Thin diagonal lines, cortex; thick diagonal lines, hippocampus; horizontal lines, cerebellum. Scale bar $=1.0 \mathrm{~mm}$. (B) Quantification of cortex, hippocampus, and cerebellum volumes. Black bars, normoxia; white bars, hypoxia. Total $n=53$ brains, $n \geq 17$ per treatment; groups balanced for sex. $* p<0.05 v s$ normoxic. Error bars $=$ SEM.

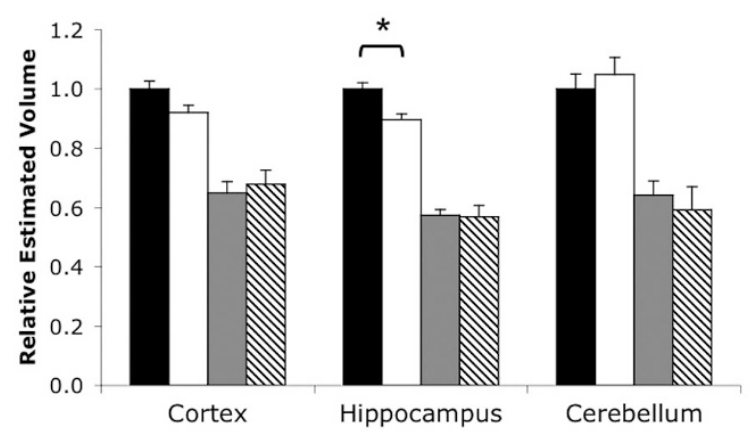

Figure 2. Male hippocampus undergoes greater volume loss compared with female. Sex differences in regional brain volumes of hypoxic and normoxic mice. Black bars, normoxic males; white bars, normoxic females; gray bars, hypoxic males; hatched bars, hypoxic females. Total $n=53$ brains, $n \geq 8$ per group. All hypoxic volumes are significantly smaller $\left({ }^{*} p<0.05\right)$ than the normoxic volumes. Error bars = SEM.

taken using a Leica DFC280 camera on a Leica stereoscope using uniform settings. Unmanipulated images were quantified using Adobe Photoshop Software. For these analyses, the brain was either examined as a whole or subdivided into five regions using anatomical markers as guides. Image intensity was inverted, a specific region was selected (lasso tool), and mean pixel intensity of this region was measured (histogram function) to estimate myelin intensity. Statistical analysis was performed using two-way ANOVA with Bonferroni post hoc comparison for whole brain measurements and three-way ANOVA with Tukey's correction for multiple comparisons for regional analyses.

Proliferation. Cell proliferation was assayed by phospho-Histone $\mathrm{H} 3$ (pHH3) immunohistochemistry. Serially cut sections on slides underwent antigen retrieval. Sections were rehydrated in PBS $(0.1 \mathrm{M})$ for $5 \mathrm{~min}$ and then placed in boiling sodium citrate $(10 \mathrm{mM})$ for $45 \mathrm{~min}$. After cooling, sections were washed, blocked in $2 \%$ normal goat serum with $0.05 \%$ triton $\mathrm{X}-100$ for $40 \mathrm{~min}$, and rabbit anti-pHH3 (1:800; Upstate) applied at $4^{\circ} \mathrm{C}$ overnight. After rinsing, biotinylated anti-rabbit $\operatorname{IgG}$ (1:500; Vector Labs) was added for 30 min followed by Vectastain ABC kit and DAB (Vector Labs) per manufacturer's instructions. Sections were counterstained with Hematoxylin QS (Vector Labs). Immunostaining was visualized on a Nikon E800 microscope. All brown cells within the hippocampus across serially cut brain sections were counted for each animal. Counts were performed blind to treatment and sex. Statistical analysis was performed using two-way ANOVA with Bonferroni post hoc comparison.

\section{RESULTS}

Hypoxia leads to differential reduction of regional brain volumes. Chronic sublethal hypoxia treatment of postnatal mouse pups greatly reduced overall brain size when measured immediately after the removal from treatment at postnatal day 11 (Fig. 1A), as anticipated from prior reports $(9,10)$. This reduction in total brain size is accompanied with overall body size reduction: hypoxic mice have a body weight reduction of $57 \pm 1.6 \%$ compared with controls, whereas brain weight is only reduced by $33 \pm 2.4 \%$. This observation is consistent with previous studies in rats (10). We found no significant difference in the overall hypoxia-induced decrease of body or brain weights when sexes were compared.

To determine whether the growth of different regions of the brain are affected differentially by hypoxia treatment, we measured the volumes of three brain regions - the cortex, hippocampus, and cerebellum - all of which are potentially affected in preterm infants. Using the Cavalieri technique (19), we measured a statistically significant reduction in all regional volumes (Fig. $1 B$; cortex: $p<0.0001, F=78.42, \mathrm{DFn}=1, \mathrm{DFd}=49$; hippocampus: $p<0.0001, F=233.6$, DFn $=1$, DFd $=$ 
A
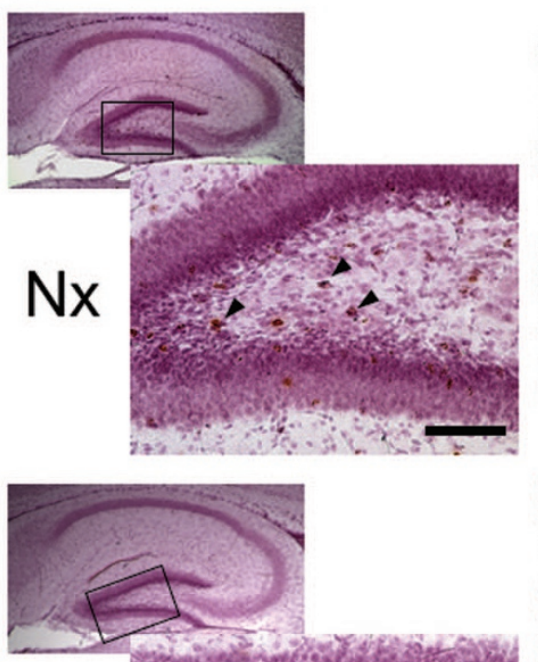

$\mathrm{Hx}$

\section{$\mathrm{B}$}
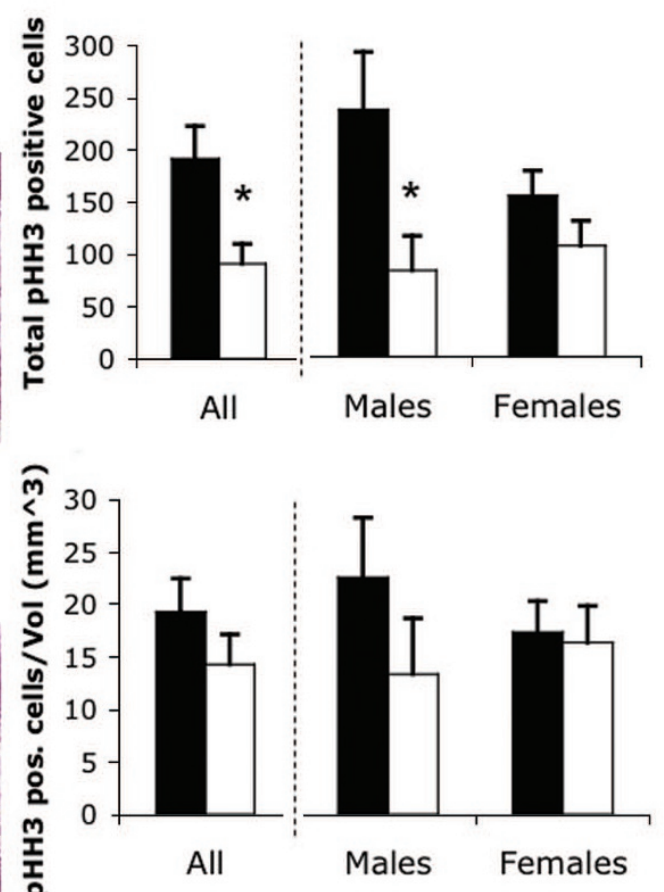

Figure 3. Hypoxia reduces absolute but not volume-relative hippocampal progenitor proliferation. (A) PHH3 immunostaining (brown cells, arrowheads) in the dentate gyrus of normoxic (Nx) and hypoxic (Hx) hippocampus. Box shows region of outset. Scale bar $=$ $100 \mu \mathrm{m}$. (B) Total numbers of $\mathrm{pHH} 3$ positive cells. (C) Total numbers of pHH3 positive cells per volume. Black bars, normoxia; white bars, hypoxia. Total $n=23$ brains, $n \geq 5$ per group. ${ }^{*} p<0.05$. Error bars $=$ SEM.
49; cerebellum: $p<0.0001, F=92.25, \mathrm{DFn}=1, \mathrm{DFd}=42$ ). Regional volumes were normalized relative to controls in each experimental set to minimize any histologic processing related volume changes. A total of 53 mouse brains were measured. Total hippocampal and cerebellar volumes were reduced by $40 \%$ each after $8 \mathrm{~d}$ of hypoxia (Fig. $1 B$, middle and right, respectively). Cortical volume was reduced by $31 \%$ (Fig. $1 B$, left).

Hypoxia abolishes normal sex differences observed in the hippocampus. We next compared regional volumes by sex and treatment. Most notably, male hippocampal volume in control pups was $10.5 \%$ larger than females $(p<0.01, F=$ 4.92, DFn $=1$, DFd = 49; Fig. 2, middle). This sex difference was abolished by hypoxia (Fig. 2, middle). Male mice treated with hypoxia had a $43 \%$ overall reduction of hippocampal volume, whereas females had a $36 \%$ reduction [difference of the means calculations, $p<0.05$; $(21)]$. Although this difference is small, it was reproduced consistently across multiple, independent experimental runs. Thus, males experience significantly greater hippocampal volume loss due to hypoxia than females.

In other brain regions, there were no significant differences in normal volumes. P11 control males seemed to have larger cortical volumes but this difference was not significant $(p>$ $0.05, F=0.55, \mathrm{DFn}=1, \mathrm{DFd}=49$; Fig. 2, left). Cerebellar volume showed no sex-linked difference $(p>0.05, F=$ 2.01e-4, DFn = 1, DFd = 42; Fig. 2, right). Therefore, despite the striking reductions in cortical and cerebellar volumes under hypoxic conditions, there are no measurable sex-linked decrements in these regions.

Hypoxia reduces total number of proliferating cells but not proliferation relative to volume in the hippocampus. We next examined cell division in normoxic and hypoxic hip- pocampi. Phospho-Histone H3 (pHH3), a mitosis marker, was used to visualize proliferating cells. Twenty-three mouse brains were analyzed. Almost all of the pHH3-positive cells were in the dentate gyrus, the primary hippocampal region of ongoing neurogenesis (Fig. 3A). We found significantly fewer total pHH3-positive cells in hypoxic hippocampus but only in males (Fig. $3 B ; p<0.05, F=6.12$, DFn $=1, \mathrm{DFd}=19$ ). However, when adjusted for hippocampal volume, there was no significant change in proliferation per unit volume (Fig. $3 C ; p=0.29, F=1.14$, DFn $=1, \mathrm{DFd}=19$ ).

Disruption of myelin formation in the brain after hypoxia treatment is sex and region dependent. BGII staining was used to visualize myelin fibers in anatomically matched medial parasagittal sections (Fig. 4A). Myelination is grossly disrupted by sublethal hypoxia (9), consistent with the white matter delays seen in preterm infants $(22,23)$. Thirteen mouse brains were measured. BGII revealed heavily myelinated tracts in hindbrain, midbrain, and cerebellum at P11 with lighter myelin staining surrounding the hippocampus and corpus callosum (Fig. $4 A$ ). BGII staining was visibly reduced in hypoxic brains, suggesting a disruption in myelination (Fig. 4A).

Quantification of BGII staining intensity in whole brain sections demonstrated significant hypomyelination in hypoxic male brains (Fig. $4 B$; $p<0.05, F=7.26$, DFn $=1, \mathrm{DFd}=$ $9)$. No statistically significant myelination differences were seen between normoxic and hypoxic females (Fig. 4B), likely due in large part to the tendency of normal P11 female brains to have less myelin than normal males.

Subdividing the brain into five regions revealed that the myelination changes were not only sex dependent but also regionally distinct. Region 1, containing cortex, hippocampus, and corpus callosum, was the only region where myelination 


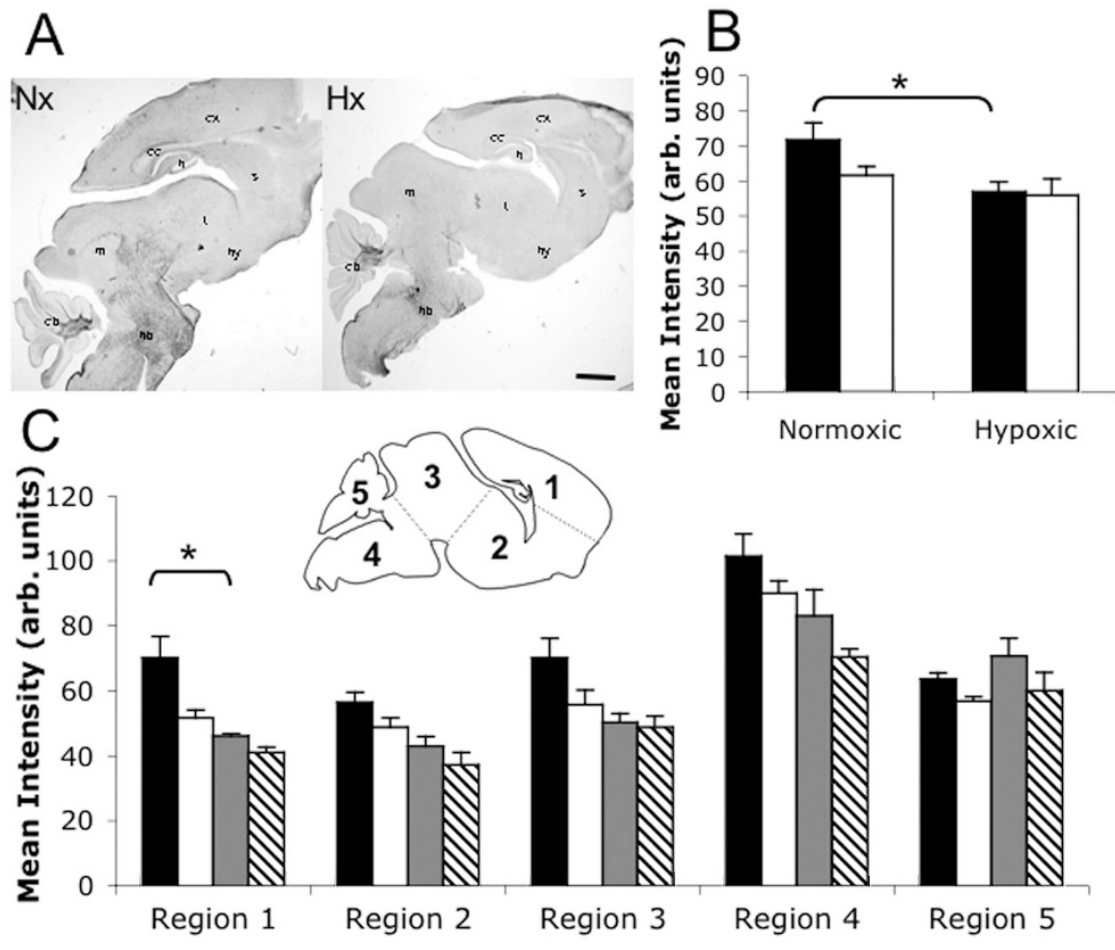

Figure 4. Chronic neonatal hypoxia produces male forebrain hypomyelination. Myelin staining in normoxic and hypoxic brains as visualized with Black Gold II. (A) BGII staining. cx, cortex; cc, corpus callosum; h, hippocampus; s, striatum; t, thalamus; hy, hypothalamus; m, midbrain; hb, hindbrain; cb, cerebellum. (B) Quantification of myelin staining intensity in whole brains. Black bars, males; white bars, females. (C) Quantification of myelin staining intensity in five labeled regions (inset image). Black bars, normoxic males; white bars, normoxic females; gray bars, hypoxic males; hatched bars, hypoxic females. Total $n=13$ brains, $n \geq 3$ per group. ${ }^{*} p<0.05$. Scale bar $=1 \mathrm{~mm}$. Error bars $=$ SEM. in males showed a statistically significant disruption (Fig. $4 C$, left; $p<0.05, F=4.73$, DFn $=4$, DFd $=45$ ). Therefore, forebrain was the main region contributing to the overall decrease in myelination observed in males. Midbrain/ hindbrain areas defined as Regions 2-4 showed a similar pattern of reduced myelination, particularly in males, but this was not significant (Fig. 4C, middle). Myelination is not affected in Region 5, cerebellum, which is consistently heavily myelinated by this stage (Fig. $4 C$, right).

\section{DISCUSSION}

The increased male susceptibility to preterm brain damage is poorly understood. Using a hypoxia model that recapitulates the neurologic pathology of premature birth $(9,10)$, we have demonstrated significantly greater hippocampal volume loss in male mice compared with females plus associated hypomyelination. This sex difference was likely not noted previously because hypoxia results in male and female brains that are comparable in size, both globally and regionally. It is only when the regional sex differences in normal brain anatomy are noted that this differential loss is revealed. Our results highlight the importance of examining sex differences in injury response in the context of differences that exist before injury.

The neonatal chronic hypoxia model is appealing because it mimics the ongoing low-grade hypoxia associated with the chronic lung disease of prematurity. Although many animal models are used to study the effects of prematurity, most suffer from expense, limited genetic tractability or poor imitation of the physiologic state of a preterm infant (24). Rodents, which are amenable to genetic manipulation and behavioral testing, do not survive preterm delivery but are born less mature (13), and so many neurologic events that are fetal in humans can be manipulated postnatally in mice. The Rice-Vannucci neonatal hypoxic-ischemic model is by far the most common model for studying neuroprotection in the developing brain (25). Additional models of ischemia or excitotoxicity have also been used (26,27). Preterm infants, however, rarely suffer acute ischemic or excitotoxic events but rather have ongoing global compromise similar to what occurs in the hypoxia model. Also, in addition to the chronic hypoxia, the mice in our study may have nutritional losses, similar to the impaired nutrition of preterm newborns. Using a foster dam paradigm to control for poor feeding or milk supply in the hypoxic mother, it was previously shown that rat pups exposed to chronic neonatal hypoxia showed poor growth despite the availability of adequate nutrition (10). Affects on pup behavior cannot be ruled out. Our foster dam addition is a variation on the prior work and likely insures adequate nutritional supplies. Thus, the majority of the volume loss is likely secondary to the hypoxia itself, even if malnutrition plays some role. Whether due to hypoxia alone or a mixture of insults, the global neurologic damage frequently seen in preterm infants, including gray and white matter loss leading to ex vacuo ventriculomegaly is closely mimicked.

Cortex, hippocampus, and cerebellum are not usually considered sexually dimorphic, but sex-linked differences are seen $(28,29)$. To the best of our knowledge, sex-linked volume differences in neonatal mouse brain regions have not been previously reported, but magnetic resonance imaging in adult mice showed a $2.5 \%$ sex-linked volume difference, with male brain being larger than female (29). Adult male C57BL/6 mice have a larger posterior hippocampus, whereas females have a larger anterior hippocampus (29). We measured the entire hippocampal volume, suggesting either that total male hippocampal volume is proportionately larger in young mice or that the posterior difference dominates the measurement. Un- 
like mice, human volumetric studies have been variable (30). Male brain volumes are generally larger but no consistent sex-related hippocampal differences have been seen (30). Differences in regional brain volumes may be species specific or may reflect different regional developmental trajectories. Although human anatomical differences may be small, compromised hippocampal function is frequently associated with volume loss in children who were born preterm $(31,32)$. Long-term behavioral studies in both mice and humans are needed to assess the contribution of sex-linked injury to functional hippocampal impairment.

All regions had volume loss with hypoxia, but hippocampal and cerebellar loss were greater than cortical loss, potentially due to developmental differences: cortical neurogenesis is essentially complete in postnatal mice, whereas the hippocampus and cerebellum are still regions of active neural proliferation. However, unlike hippocampus, no sex volume difference was detected in cerebellum nor did cerebellar myelination change with hypoxia.

Given the differential reductions in hippocampal volumes, we assessed progenitor proliferation in this region. In rats, hypoxia significantly disrupts neural proliferation (33), although reduced neuron size, defects in differentiation, and increased apoptosis also result from hypoxic injury $(11,33)$. The role of sex in neurogenesis remains uncertain. For example, estrogen has variable effects on dentate gyrus neurogenesis $(34,35)$. Despite the striking reduction in proliferating cells in hypoxia, there was no statistical decrease in proliferation when normalized for total volume changes. This finding suggests that differential reductions in cell division are not the sole cause of volume loss.

Previous work in this model suggested suppression of glial genes suggesting a role for gliogenesis (9). Adult males have more white matter than females $(36,37)$. This difference is less pronounced in our normal neonatal mice but seems to be developing by P11. Similar to the difference in hippocampal volume, the decrease in forebrain myelination seen in hypoxic neonatal males may be due to tendency to have a relatively greater normal male baseline. Other brain regions showed smaller decreases in myelin intensity after hypoxia. Differential maturation of glia may underlie these regional differences (38), a hypothesis that requires further investigation with oligodendrocyte lineage markers. Determining whether the hypoxia-induced hypomyelination in males can be averted or reversed using sex steroids or other agents is critical.

Sexually dimorphic responses to disease or injury have long been recognized. For example, women have a lower risk of stroke and greater recovery, an effect attributed primarily to sex steroids. In vitro evidence shows that estrogen can be neuroprotective in response to hypoxia (39) and oxygenglucose deprivation (40), whereas dihydrotestosterone (a "male" hormone) was found to increase muscimol-induced excitotoxic cell death (41). In vivo evidence on sex steroid protection is far more variable, varying with age, insult, brain region, and hormone examined (42-44). Sex hormone level differences in neonatal male and female mice are well established, with males having a neonatal testosterone surge and females an estrogen surge. We have preliminary data that suggests that these hormones are altered differentially in males and females in response to hypoxia treatment (data not shown), but the impact of any alterations is the subject of ongoing investigation. Estrogen and progesterone supplementation has been tried in a small cohort of preterm females (45), but the consequences of this treatment remain uncertain, especially if this approach were to be expanded to males. The complexity and variability underscores the need to assess the effects of these hormones in vivo in a model that appropriately mimics the specific type of damage in question.

Recent studies also suggest sex-specific differences in neuroprotective or apoptotic gene expression $(43,44)$. One intriguing study (46) showed that one could predict a $1 / 3$ lower mortality in females due to hypoxic events if there existed a protective X-linked allele. The identity of the allele that could lower female risk of death remains elusive, but there are hints that there may be differences in the developmental expression of apoptotic pathways $(43,44)$. Using an acute ischemic challenge, male neurons demonstrate greater caspase-independent, apoptosis-inducing factor (AIF)-dependent cell death compared with female neurons (44). The potential roles of differential use of specific apoptotic pathways and the underlying influence of $\mathrm{X}$ - and Y-linked gene expression in the setting of chronic hypoxia can now be assessed at a mechanistic level using the multiple genetic tools available in mice.

This study is limited primarily by our ability to detect small but potentially significant, sex-specific anatomical changes beyond the hippocampus. 3D-MRI analysis of the brains of normal and hypoxia-treated neonatal mice might prove revealing because differences of as small as $2.5 \%$ could be detected in adult mice (29). Although body weight rapidly recovers after chronic hypoxia, brain volume does not recover [(11); unpublished observations], despite the increase in cell proliferation in the weeks after hypoxia. Noninvasive measurements of the specific trajectories of gray and white matter damage and recovery after hypoxia in each sex would likely be revealing.

The increased loss of male hippocampal volume after chronic postnatal hypoxia suggests that we will see sex differences in standardized tests of rodent behavior. Determining the link between the anatomical losses and behavioral impact of these changes will be critical in assessing new interventions aimed at ameliorating these losses. Being able to mimic male susceptibility to prematurity-associated neurologic damage provides a new way to investigate the biologic basis of this damage and to rapidly test new neuroprotective strategies.

Acknowledgments. We thank L.R. Ment and M. Schwartz for their guidance in setting up the hypoxia model system. We thank DL Kimmel for image data quantification and statistical help. We thank all members of the Penn Lab and Ron Wong for close reading of the manuscript.

\section{REFERENCES}

1. Martin JA, Hamilton BE, Sutton PD, Ventura SJ, Menacker F, Kirmeyer S, Munson ML; Centers for Disease Control and Prevention National Center for Health Statistics National Vital Statistics System 2007 Births: final data for 2005. Natl Vital Stat Rep 56:1-103 
2. Anderson PJ, Doyle LW 2008 Cognitive and educational deficits in children born extremely preterm. Semin Perinatol 32:51-58

3. Marlow N, Wolke D, Bracewell MA, Samara M 2005 Neurologic and developmental disability at six years of age after extremely preterm birth. N Engl J Med 352:9-19

4. Chyi LJ, Lee HC, Hintz SR, Gould JB, Sutcliffe TL 2008 School outcomes of late preterm infants: special needs and challenges for infants born at 32 to 36 weeks gestation. J Pediatr 153:25-31

5. Petrou S, Henderson J, Bracewell M, Hockley C, Wolke D, Marlow N 2006 Pushing the boundaries of viability: the economic impact of extreme preterm birth. Early Hum Dev 82:77-84

6. Thompson DK, Warfield SK, Carlin JB, Pavlovic M, Wang HX, Bear M, Kean MJ, Doyle LW, Egan GF, Inder TE 2007 Perinatal risk factors altering regional brain structure in the preterm infant. Brain 130:667-677

7. Kesler SR, Ment LR, Vohr B, Pajot SK, Schneider KC, Katz KH, Ebbitt TB, Duncan CC, Makuch RW, Reiss AL 2004 Volumetric analysis of regional cerebral development in preterm children. Pediatr Neurol 31:318-325

8. Hart AR, Whitby EW, Griffiths PD, Smith MF 2008 Magnetic resonance imaging and developmental outcome following preterm birth: review of current evidence. Dev Med Child Neurol 50:655-663

9. Curristin SM, Cao A, Stewart WB, Zhang H, Madri JA, Morrow JS, Ment LR 2002 Disrupted synaptic development in the hypoxic newborn brain. Proc Natl Acad Sci USA 99:15729-15734

10. Ment LR, Schwartz M, Makuch RW, Stewart WB 1998 Association of chronic sublethal hypoxia with ventriculomegaly in the developing rat brain. Brain Res Dev Brain Res 111:197-203

11. Schwartz ML, Vaccarino F, Chacon M, Yan WL, Ment LR, Stewart WB 2004 Chronic neonatal hypoxia leads to long term decreases in the volume and cell number of the rat cerebral cortex. Semin Perinatol 28:379-388

12. Stewart WB, Ment LR, Schwartz M 1997 Chronic postnatal hypoxia increases the numbers of cortical neurons. Brain Res 760:17-21

13. Clancy B, Kersh B, Hyde J, Darlington RB, Anand KJ, Finlay BL 2007 Web-based method for translating neurodevelopment from laboratory species to humans. Neuroinformatics 5:79-94

14. Fagel DM, Ganat Y, Silbereis J, Ebbitt T, Stewart W, Zhang H, Ment LR, Vaccarin FM 2006 Cortical neurogenesis enhanced by chronic perinatal hypoxia. Exp Neurol 199:77-91

15. Turner CP, Seli M, Ment L, Stewart W, Yan H, Johansson B, Fredholm BB, Blackburn M, Rivkees SA 2003 A1 adenosine receptors mediate hypoxia-induced ventriculomegaly. Proc Natl Acad Sci USA 100:11718-11722

16. Fagel DM, Ganat Y, Cheng E, Silbereis J, Ohkubo Y, Ment LR, Vaccarino FM 2009 Fgfrl is required for cortical regeneration and repair after perinatal hypoxia J Neurosci 29:1202-1211

17. McClive PJ, Sinclair AH 2001 Rapid DNA extraction and PCR-sexing of mouse embryos. Mol Reprod Dev 60:225-226

18. Jimenez A, Fernandez R, Madrid-Bury N, Moreira PN, Borque C, Pintado B, Gutierrez-Adan A 2003 Experimental demonstration that pre- and post-conceptional mechanisms influence sex ratio in mouse embryos. Mol Reprod Dev 66:162-165

19. Howard V, Reed M 1998 Unbiased stereology: Three-Dimensional Measurement in Microscopy. Springer, New York, p 246

20. Schmued L, Bowyer J, Cozart M, Heard D, Binienda Z, Paule M 2008 Introducin Black-Gold II, a highly soluble gold phosphate complex with several unique advantages for the histochemical localization of myelin. Brain Res 1229:210-217

21. Matthews JN, Altman DG 1996 Statistics notes. Interaction 2: compare effect sizes not $\mathrm{P}$ values. BMJ 313:808

22. Constable RT, Ment LR, Vohr BR, Kesler SR, Fulbright RK, Lacadie C, Delancy S, Katz KH, Schneider KC, Schafer RJ, Makuch RW, Reiss AR 2008 Prematurely born children demonstrate white matter microstructural differences at 12 years of age, relative to term control subjects: an investigation of group and gender effects. Pediatrics 121:306-316
23. Edgin JO, Inder TE, Anderson PJ, Hood KM, Clark CA, Woodward LJ 2008 Executive functioning in preschool children born very preterm: relationship with early white matter pathology. J Int Neuropsychol Soc 14:90-101

24. Elovitz MA, Mrinalini C 2004 Animal models of preterm birth. Trends Endocrinol Metab 15:479-487

25. Rice JE 3rd, Vannucci RC, Brierley JB 1981 The influence of immaturity on hypoxic-ischemic brain damage in the rat. Ann Neurol 9:131-141

26. Derugin N, Ferriero DM, Vexler ZS 1998 Neonatal reversible focal cerebral ischemia: a new model. Neurosci Res 32:349-353

27. Nunez JL, Alt JJ, McCarthy MM 2003 A new model for prenatal brain damage. I. GABAA receptor activation induces cell death in developing rat hippocampus. Exp Neurol 181:258-269

28. Gur RC, Turetsky BI, Matsui M, Yan M, Bilker W, Hughett P, Gur RE 1999 Sex differences in brain gray and white matter in healthy young adults: correlations with cognitive performance. J Neurosci 19:4065-4072

29. Spring S, Lerch JP, Henkelman RM 2007 Sexual dimorphism revealed in the structure of the mouse brain using three-dimensional magnetic resonance imaging. Neuroimage 35:1424-1433

30. Cosgrove KP, Mazure CM, Staley JK 2007 Evolving knowledge of sex differences in brain structure, function, and chemistry. Biol Psychiatry 62:847-855

31. Beauchamp MH, Thompson DK, Howard K, Doyle LW, Egan GF, Inder TE, Anderson PJ 2008 Preterm infant hippocampal volumes correlate with later working memory deficits. Brain 131:2986-2994

32. Isaacs EB, Lucas A, Chong WK, Wood SJ, Johnson CL, Marshall C, VarghaKhadem F, Gadian DG 2000 Hippocampal volume and everyday memory in children of very low birth weight. Pediatr Res 47:713-720

33. Robinson S, Petelenz K, Li Q, Cohen ML, Dechant A, Tabrizi N, Bucek M, Lust D, Miller RH 2005 Developmental changes induced by graded prenatal systemic hypoxic-ischemic insults in rats. Neurobiol Dis 18:568-581

34. Galea LA 2008 Gonadal hormone modulation of neurogenesis in the dentate gyrus of adult male and female rodents. Brain Res Rev 57:332-341

35. Lagace DC, Fischer SJ, Eisch AJ 2007 Gender and endogenous levels of estradiol do not influence adult hippocampal neurogenesis in mice. Hippocampus 17:175-180

36. De Bellis MD, Keshavan MS, Beers SR, Hall J, Frustaci K, Masalehdan A, Noll J, Boring AM 2001 Sex differences in brain maturation during childhood and adolescence. Cereb Cortex 11:552-557

37. Yang S, Li C, Zhang W, Wang W, Tang Y 2008 Sex differences in the white matte and myelinated nerve fibers of Long-Evans rats. Brain Res 1216:16-23

38. Khwaja O, Volpe JJ 2008 Pathogenesis of cerebral white matter injury of prematurity. Arch Dis Child Fetal Neonatal Ed 93:F153-F161

39. Heyer A, Hasselblatt M, von Ahsen N, Hafner H, Siren AL, Ehrenreich H 2005 In vitro gender differences in neuronal survival on hypoxia and in 17beta-estradiolmediated neuroprotection. J Cereb Blood Flow Metab 25:427-430

40. Liu M, Hurn PD, Roselli CE, Alkayed NJ 2007 Role of P450 aromatase in sex-specific astrocytic cell death. J Cereb Blood Flow Metab 27:135-141

41. Nunez JL, McCarthy MM 2008 Androgens predispose males to GABAA-mediated excitotoxicity in the developing hippocampus. Exp Neurol 210:699-708

42. Wagner CK 2008 Progesterone receptors and neural development: a gap between bench and bedside? Endocrinology 149:2743-2749

43. Nunez JL, McCarthy MM 2003 Sex differences and hormonal effects in a model of preterm infant brain injury. Ann N Y Acad Sci 1008:281-284

44. Renolleau S, Fau S, Charriaut-Marlangue C 2008 Gender-related differences in apoptotic pathways after neonatal cerebral ischemia. Neuroscientist 14:46-52

45. Trotter A, Pohlandt F 2000 The replacement of oestradiol and progesterone in very premature infants. Ann Med 32:608-614

46. Mage DT, Donner M 2006 Female resistance to hypoxia: does it explain the sex difference in mortality rates? J Womens Health (Larchmt) 15:786-794 